\title{
Prognostic Value of Terminal Ileal Inflammation in Patients with Ulcerative Colitis
}

\author{
Jongwook Yu, Jihye Park, Eun Ae Kang, Soo Jung Park, Jae Jun Park, Tae II Kim, Won Ho Kim, and Jae Hee \\ Cheon \\ Department of Internal Medicine and Institute of Gastroenterology, Yonsei University College of Medicine, Seoul, Korea
}

\section{Article Info}

Received September 21, 2020

Revised November 19, 2020

Accepted December 18, 2020

Corresponding Author

Jae Hee Cheon

ORCID https://orcid.org/0000-0002-2282-8904

E-mail Geniushee@yuhs.ac
Background/Aims: Few studies have investigated terminal ileal lesions and their prognostic value in patients with ulcerative colitis (UC). We evaluated the clinical significance of these lesions as a prognostic factor in patients with UC who were in clinical remission.

Methods: We retrospectively selected 567 of 4,066 colonoscopy reports that included positive findings from orificial observations of the terminal ileum $(\mathrm{TI})$ and appendix in patients with UC. We finally recruited patients who were in clinical remission $(n=204)$. We compared the clinical courses, including relapse and other prognostic parameters associated with UC, between the groups.

Results: The baseline patient characteristics were not significantly different between patients with ( $n=69, \mathrm{TI}+$ group) and without $\mathrm{TI}$ lesions ( $\mathrm{n}=135, \mathrm{TI}-$ group), although there were more never-smokers in the $\mathrm{TI}+$ group $(n=57$ [82.6\%] in the TI+ group; $n=86$ [63.7\%] in the TI- group; $\mathrm{p}=0.005$ ). Of note, appendiceal orifice inflammation (AOI) was less frequently found in the $\mathrm{TI}+$ group (14.5\%) than in the TI- group $(71.9 \%, p<0.001)$. The cumulative relapse rate was numerically higher in the TI- group, but it was not significantly different according to the Kaplan-Meier analysis $(p=0.116)$. Multivariate Cox regression analysis also revealed advanced age at diagnosis as the most significant factor (adjusted hazard ratio, 0.964; 95\% confidence interval, 0.932 to $0.998 ; p=0.037$ ), but neither $\mathrm{TI}$ inflammation nor $\mathrm{AOI}$ were significantly associated with the cumulative relapse rate in patients with $\mathrm{UC}$ in clinical remission.

Conclusions: For patients with UC in clinical remission, neither terminal ileal lesions nor AOI had significant clinical or predictive value for future relapse. (Gut Liver 2021;15:858-866)

Key Words: Appendix; Ileum; Inflammation; Inflammatory bowel disease; Ulcerative colitis

\section{INTRODUCTION}

Ulcerative colitis (UC) is a chronic relapsing inflammatory bowel disease characterized by repeated bloody diarrhea, tenesmus, abdominal pain, and various extraintestinal manifestations. The etiology of UC has not been fully elucidated; nevertheless, an interplay of genetic, immunological, and environmental etiologies has been suggested so far. ${ }^{1,2}$

The ultimate goal of UC management is the induction and maintenance of clinical and endoscopic remission. Conventional treatment, including anti-inflammatory agents such as 5-aminosalicylic acid or corticosteroids and immunosuppressive agents such as azathioprine, 6-mer- captopurine or methotrexate, is the cornerstone of UC management. Biologic therapy is required for patients who are intolerant or refractory to the conventional treatment. ${ }^{3}$ Initially, UC involves the rectum, and progressively extends proximally. The proximal disease extension was reported to have a poor prognosis, including an increased incidence of relapse and hospitalization.,

It is important to assess prognostic predictors for disease progression to improve the management, given the relapsing-remitting course of UC. In patients with an early stage of inflammatory bowel disease, controlling both symptoms and inflammation improves future outcomes and reduces the risk of end-organ damage, such as the occurrence of stricture, fistula, and functional impairment. ${ }^{6}$ 
Late onset, early mucosal healing, low disease extent, few medications, and cigarette smoking are known as favorable prognostic factors of UC. . $^{7-11}$

Appendiceal orifice inflammation (AOI) is commonly found in patients with UC, and has been studied for its possible association with the pathophysiology and prognosis of UC. The clinical significance of AOI in patients with UC has not been definitely concluded; however, its association with the pathogenesis or prognosis of UC is unlikely. ${ }^{12-15}$

On the other hand, during routine ileo-colonoscopy, incidental terminal ileal lesions are commonly found and known to have no significant prognostic implications in the healthy population. ${ }^{16-20}$ Similarly, these terminal ileum (TI) findings have been reported in patients with UC; however, few studies have investigated the prognostic significance of these incidental findings in patients with UC. Moreover, the possible association of these TI findings with the pathophysiology of UC has not yet been investigated. ${ }^{21}$

Therefore, we performed this retrospective study to evaluate TI inflammation as a possible clinical and prognostic factor for future clinical relapse in patients with UC in clinical remission.

\section{MATERIALS AND METHODS}

\section{Study population}

This study was a retrospective observational study in a single tertiary center (Severance Hospital, Yonsei University, Seoul) in Korea. We retrospectively reviewed the medical records (full colonoscopic examination reports) of patients who were diagnosed with UC from November 2005 to February 2020 based on clinical, radiological, endoscopic, and histologic findings. ${ }^{22}$

\section{Ethical considerations}

This study was performed in accordance with the ethical guidelines of the 1975 Declaration of Helsinki, and was approved by the Institutional Review Board of Severance Hospital (IRB number: 4-2020-0696). The informed consent was waived.

\section{Data collection}

We retrospectively analyzed the clinical and endoscopic data of patients with UC. Although the data were collected retrospectively from electronic medical charts and colonoscopic reports, all variables were prospectively recorded for per-patient analysis. The enrolled patients with UC were those who experienced their first colonoscopic examina- tions after achieving clinical remission. At baseline, we investigated the patients' age, age at diagnosis, sex, smoking history, surgical history (including a history of appendectomy), height, weight, body mass index, disease extent at diagnosis, age at the time of remission of colonoscopy, and the time from diagnosis to remission. We also collected the data at the time of clinical remission and relapse. We used the Montreal classification, Mayo score, and partial Mayo score to standardize the patients' disease activity. We evaluated each patient's medical records (with respect to hospitalization, medication history, date of relapse, last outpatient visit, or death) to analyze the cumulative events.

\section{Classification and definition}

1) Definitions of clinical remission and clinical relapse

Clinical disease activity was assessed by the Mayo score (0-12 points). If colonoscopy could not be performed, we assessed clinical disease activity with partial Mayo score (0-9 points). Although the noninvasive partial Mayo score does not include endoscopic assessment, it reflects the clinical disease activity as accurately as the full Mayo score. ${ }^{23}$ Clinical remission was defined as a Mayo score of 2 points or less, and mucosal healing was defined as a Mayo endoscopic score of 1 or $0 .{ }^{24}$

In this study, we screened the patients who experienced a full colonoscopic examination, including TI or appendiceal orifice lesions. Most colonoscopic examinations were the first colonoscopies after achieving remission to evaluate treatment response and mucosal status. Terminal ileal or appendix orifice inflammation were defined as one or a few small-sized focal mucosal inflammatory lesions surrounded by the normal mucosa, or regenerating epithelium covered by thin or faint exudates in the TI or appendix orifice. ${ }^{20,25,26}$ Then, we calculated the Mayo score to evaluate the disease activity. Finally, we recruited patients $(n=204)$ who were in clinical remission (Mayo score of 2 points and less) with mucosal healing on colonoscopy (Mayo endoscopic score 0 and 1). . $3,27-32^{2}$

We followed the clinical courses of patients through review of medical records and searched for events of clinical relapse. In all cases of exacerbation, we defined disease relapse as a Mayo score of $\geq 6$ (partial Mayo score $\geq 5$, if colonoscopy was not performed) with any of the following: any treatment escalation during acute exacerbation; prescription of high-dose corticosteroids to control an acute UC flare; and need for hospitalization due to an exacerbation of UC. ${ }^{33}$ We used the full Mayo score if colonoscopy was performed, and partial Mayo score if colonoscopy was not possible in an acute disease flare. If there were other definite causes for exacerbation, such as acute gastroenteritis, infectious colitis such as cytomegalovirus colitis, or 
irritable bowel syndrome, they were not defined as relapses of UC.

\section{2) Assessment of the extent of disease}

We reviewed electronic medical records and endoscopic images to evaluate the extent of the disease at the time of diagnosis and study start point of UC. The disease extent was categorized into three groups using the Montreal classification. ${ }^{31,34}$

\section{Statistical analysis}

We analyzed the baseline characteristics using the Student t-test or Mann-Whitney U test for continuous variables, and the chi-square test or Fisher exact test for categorical variables. Outcomes with a normal distribution were presented as means and standard deviation, whereas non-normally distributed outcomes were presented as medians and interquartile range (IQR). The Kaplan-Meier survival analysis was used to estimate the cumulative relapse of patients according to the variables. Univariate analysis was performed using the log-rank model. Hazard ratios (HRs) and the corresponding 95\% confidence intervals (CIs) were also calculated. Multivariate analysis was performed using the Cox regression model to control for multiple risk factors that could influence relapse. Results were considered statistically significant at $p<0.05$. All statistical analyses were performed with SPSS version 20 (SPSS Inc., Chicago, IL, USA).

\section{RESULTS}

\section{Baseline patient characteristics}

Of 4,066 colonoscopic examinations performed in patients with UC, we selected reports that included TI and appendiceal orifice observations (Fig. 1). We then reviewed the medical charts and endoscopic images of 567 cases of colonoscopy, and finally recruited patients who were in clinical remission ( $\mathrm{n}=204)$. Among them, 69 patients had TI lesions, and the other 135 did not (Fig. 2).
The major baseline characteristics were not significantly different between patients with and without TI lesions. However, there were more never-smokers in TI+ group $(\mathrm{n}=57$ [82.6\%] in TI+ group; $\mathrm{n}=86$ [63.7\%] in TI- group; $\mathrm{p}=0.005$ ) (Table 1). Of note, AOI was less frequently observed in TI+ group (14.5\%) than in TI- group $(71.9 \%$, $\mathrm{p}<0.001$ ) (Table 1). In addition, there was a greater proportion of patients with a Mayo endoscopic score of 1 in TI+ group than in TI- group (58.0\% in TI+ group vs $32.6 \%$ in TI- group; $\mathrm{p}=0.001$ ).

\section{Effects of positive TI lesions on disease prognosis}

There were no significant differences in the clinical and endoscopic relapses between TI+ and TI- groups (five relapses in 69 patients [7.2\%] for TI+ group vs 20 relapses in 135 patients [14.8\%] for TI- group; $\mathrm{p}=0.119$ ). The median time from remission to relapse was 36.0 months (IQR, 12.5 to 51.0 months) for TI+ group and 31.0 months (IQR, 8.3 to 65.8 months) for TI- group ( $\mathrm{p}=0.919)$ (Table 2).

The cumulative relapse rate was higher in TI- group (Table 2), although there was no significant difference according to the Kaplan-Meier analysis $(\mathrm{p}=0.116)$ (Fig. 3 ). In addition, the cumulative incidence of hospitalization due to acute exacerbation and that of biologic agent use were not significantly different between TI+ and TIgroups ( $\mathrm{p}=0.556, \mathrm{p}=0.678$, respectively) (Fig. 3 ). Univariate

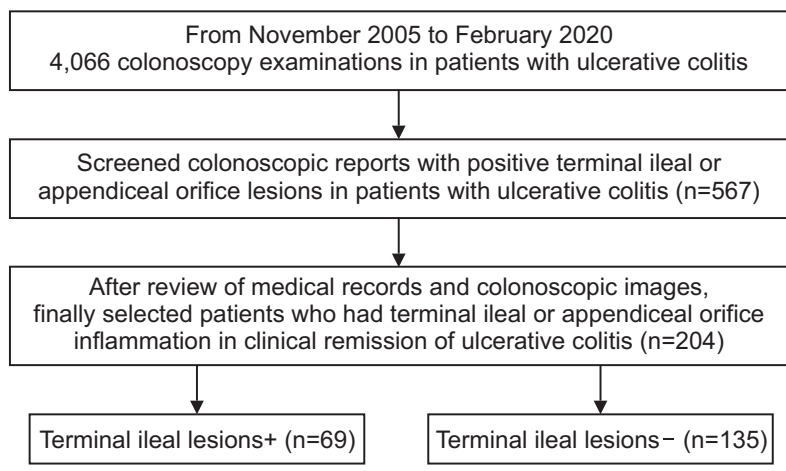

Fig. 2. Study flowchart.
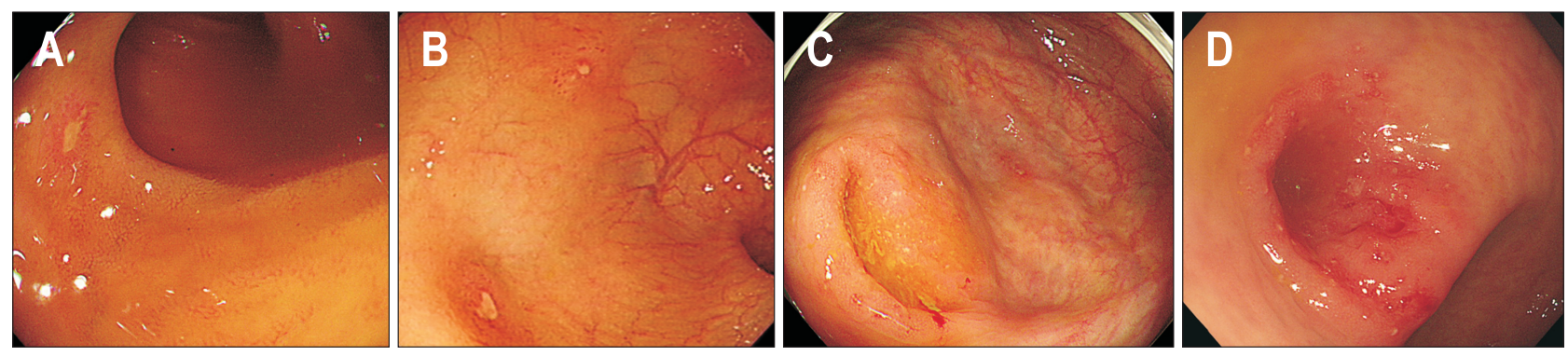

Fig. 1. Representative colonoscopy findings of terminal ileal inflammation $(A, B)$ and appendiceal orifice inflammation $(C, D)$. 
Table 1. Baseline Patient Characteristics According to Terminal Ileal Lesions

\begin{tabular}{|c|c|c|c|}
\hline \multirow{2}{*}{ Characteristics } & \multicolumn{2}{|c|}{ Terminal ileal lesion } & \multirow{2}{*}{$\mathrm{p}$-value } \\
\hline & Positive (n=69) & Negative ( $n=135$ ) & \\
\hline Age at diagnosis, yr & $36.29 \pm 14.58$ & $37.28 \pm 14.86$ & 0.651 \\
\hline Age at the time of colonoscopy on remission, yr & $42.51 \pm 13.91$ & $41.61 \pm 15.28$ & 0.682 \\
\hline Height, cm & $165.99 \pm 8.89$ & $166.73 \pm 8.97$ & 0.573 \\
\hline Weight, kg & $62.22 \pm 13.78$ & $62.60 \pm 12.36$ & 0.841 \\
\hline $\mathrm{BMI}, \mathrm{kg} / \mathrm{m}^{2}$ & $22.49 \pm 4.21$ & $22.41 \pm 3.34$ & 0.879 \\
\hline Sex & & & 0.151 \\
\hline Male & $31(44.9)$ & $75(55.6)$ & \\
\hline Female & $38(55.1)$ & $60(44.4)$ & \\
\hline Smoking history at diagnosis & & & 0.005 \\
\hline Never-smoker & $57(82.6)$ & $86(63.7)$ & \\
\hline Former smoker & $10(14.5)$ & $37(27.4)$ & \\
\hline Current smoker & $2(2.9)$ & 12 (8.9) & \\
\hline Family history & $3(4.3)$ & $11(8.1)$ & 0.310 \\
\hline Appendectomy & $3(4.3)$ & $6(4.4)$ & 0.975 \\
\hline Time from diagnosis to colonoscopy at clinical remission, mo & $63.0(37.0-109.0)$ & $38.0(21.0-66.0)$ & $<0.001$ \\
\hline Appendiceal orifice inflammation & $10(14.5)$ & 97 (71.9) & $<0.001$ \\
\hline Initial disease extent (Montreal classification) & & & 0.154 \\
\hline AOI only & 0 & $2(1.5)$ & \\
\hline E1 & $28(40.6)$ & $65(48.1)$ & \\
\hline E2 & 23 (33.3) & $42(31.1)$ & \\
\hline E3 & $18(26.1)$ & 26 (19.3) & \\
\hline \multicolumn{4}{|l|}{ Medications at remission } \\
\hline 5-ASA & $68(98.6)$ & $132(97.8)$ & 0.706 \\
\hline Azathioprine/6-MP & $10(14.5)$ & 18 (13.3) & 0.820 \\
\hline Biologic agents & 6 (8.7) & $6(4.4)$ & 0.222 \\
\hline Mayo score at remission & & & 0.705 \\
\hline $0-1$ & 59 (85.5) & $118(87.4)$ & \\
\hline 2 & $10(14.5)$ & $17(12.6)$ & \\
\hline Mayo endoscopic score & & & 0.001 \\
\hline 0 & $29(42.0)$ & $91(67.4)$ & \\
\hline 1 & $40(58.0)$ & $44(32.6)$ & \\
\hline
\end{tabular}

Data are presented as mean $\pm S D$, number $(\%)$, or median (interquartile range).

BMI, body mass index; AOI, appendiceal orifice inflammation; 5-ASA, 5-acetylsalicylic acid; 6-MP, 6-mercaptopurine.

Table 2. Relapses According to Terminal Ileal Lesions

\begin{tabular}{|c|c|c|c|}
\hline \multirow{2}{*}{ Variable } & \multicolumn{2}{|c|}{ Terminal ileal lesion } & \multirow{2}{*}{$\mathrm{p}$-value } \\
\hline & Positive $(n=69)$ & Negative ( $n=135$ ) & \\
\hline Relapse & $5(7.2)$ & 20 (14.8) & 0.119 \\
\hline Addition of biologics agents & $1(1.4)$ & $3(2.2)$ & 0.706 \\
\hline Hospitalization due to acute exacerbation & $3(4.3)$ & 8 (5.9) & 0.637 \\
\hline Time from remission to relapse, mo & $36.0(12.5-51.0)$ & $31.0(8.3-65.8)$ & 0.919 \\
\hline Mayo score at relapse (0-12) & & & 0.457 \\
\hline Moderate (6-10) & $4(80.0)$ & 12 (92.3) & \\
\hline Severe (11-12) & $1(20.0)$ & $1(7.7)$ & \\
\hline Partial Mayo score at relapse (0-9) & & & - \\
\hline Moderate (5-6) & 0 & $3(42.9)$ & \\
\hline Severe (7-9) & 0 & $4(57.1)$ & \\
\hline
\end{tabular}

Data are presented as number (\%) or median (interquartile range).

Definition of relapse: Mayo $\geq 6$; if colonoscopy was not performed, partial Mayo $\geq 5$.

analysis, using the log-rank test, of relapse showed that an advanced age at the time of UC diagnosis was significantly related to the decreased relapse rate (HR, 0.966; 95\%
CI, 0.936 to $0.998 ; \mathrm{p}=0.036$ ). Moreover, multivariate Cox regression analysis also revealed advanced age as a significant factor for relapse (adjusted HR, 0.964; 95\% CI, 0.932 
A
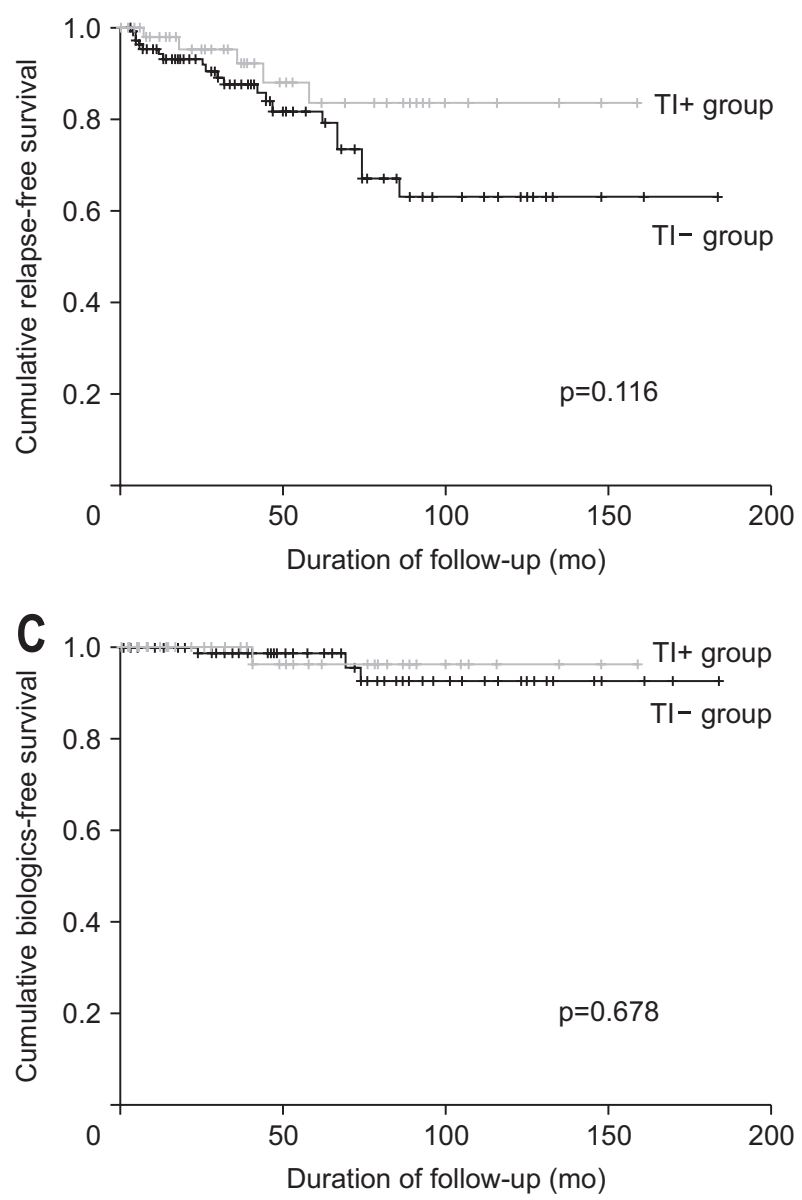

B

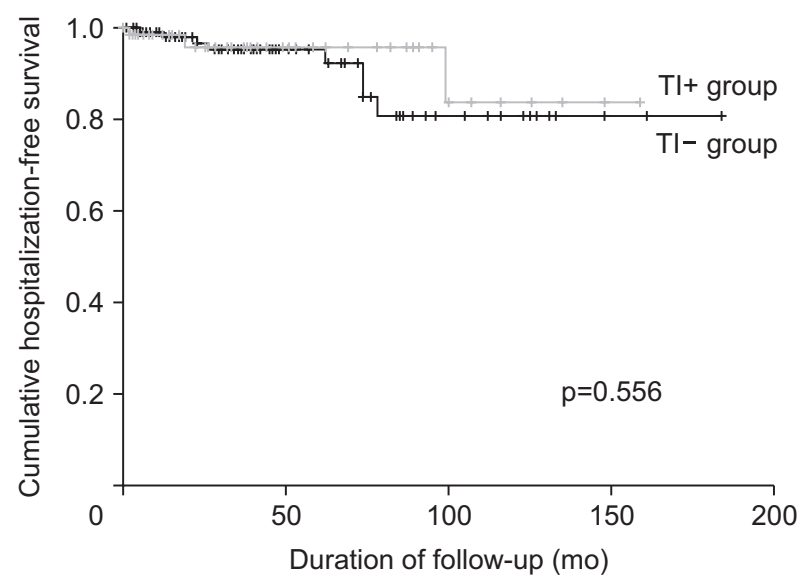

Fig. 3. Kaplan-Meier curves. (A) Cumulative relapse-free survival. (B) Cumulative hospitalization-free survival. (C) Cumulative biologicsfree survival.

TI, terminal ileum.

Table 3. Univariate and Multivariate Analyses of the Effect of Terminal Inflammation on Ulcerative Colitis for Predicting Future Relapse

\begin{tabular}{|c|c|c|c|c|}
\hline \multirow{2}{*}{ Variable } & \multicolumn{2}{|c|}{ Univariate analysis } & \multicolumn{2}{|c|}{ Multivariate analysis } \\
\hline & $\operatorname{HR}(95 \% \mathrm{Cl})$ & $\mathrm{p}$-value & Adjusted HR (95\% Cl) & $\mathrm{p}$-value \\
\hline Male & Reference & & Reference & \\
\hline Female & $1.184(0.536-2.615)$ & 0.677 & 1.449 (0.574-3.657) & 0.216 \\
\hline Age at the UC diagnosis & $0.966(0.936-0.998)$ & 0.036 & $0.964(0.932-0.998)$ & 0.037 \\
\hline E1 & Reference & & Reference & \\
\hline E2 at the diagnosis & 0.503 (0.182-1.387) & 0.184 & $0.503(0.177-1.428)$ & 0.197 \\
\hline E3 at the diagnosis & $1.123(0.427-2.951)$ & 0.814 & $1.165(0.438-3.100)$ & 0.760 \\
\hline Never-smoking & Reference & & Reference & \\
\hline Ex-smoking & $1.126(0.467-2.718)$ & 0.791 & $1.521(0.564-4.099)$ & 0.407 \\
\hline Current smoking & $0.859(0.112-6.568)$ & 0.883 & 0.999 (0.119-8.408) & 0.999 \\
\hline Family history & $1.141(0.269-4.847)$ & 0.858 & & \\
\hline History of appendectomy & $0.045(0.000-54.85)$ & 0.392 & & \\
\hline Age during colonoscopy at remission & $0.966(0.937-0.995)$ & 0.021 & & \\
\hline $\mathrm{AOI}$ on remission & $1.250(0.555-2.812)$ & 0.590 & $1.383(0.536-3.568)$ & 0.503 \\
\hline TI lesions on remission & $0.465(0.174-1.239)$ & 0.126 & 0.583 (0.197-1.729) & 0.331 \\
\hline
\end{tabular}

$\mathrm{HR}$, hazard ratio; $\mathrm{Cl}$, confidence interval; UC, ulcerative colitis; E1, ulcerative proctitis; E2, left-sided ulcerative colitis; E3, extensive ulcerative colitis; AOI, appendiceal orifice inflammation; TI, terminal ileum.

to $0.998 ; \mathrm{p}=0.037$ ) (Table 3 ). Neither TI lesions nor AOI were significantly associated with future relapse.

For the TI lesions $(n=69)$, biopsies were performed in
$53.6 \%(n=37)$. Chronic nonspecific inflammation was the most common pathologic finding (chronic nonspecific inflammation [ $\mathrm{n}=14,37.8 \%]$; lymphoid hyperplasia [ $\mathrm{n}=11$, 
29.7\%]; erosion [ $n=6,16.2 \%]$; chronic ileitis $[n=4,10.8 \%]$; necrotic inflammatory exudate $[n=1,2.7 \%])$.

\section{Effects of AOI on disease prognosis}

On the other hand, for patients who had appendiceal orifice lesions ( $\mathrm{n}=107, \mathrm{AOI}+$ group) in their remission state, more patients in AOI+ group than in AOI- group ( $n=97$, AOI- group) had a disease extent of E1 (53.3\% for $\mathrm{AOI}+$ group vs $37.1 \%$ for AOI- group; $\mathrm{p}=0.003$ ) (Table $4)$, and fewer patients had a history of appendectomy (0 for AOI+ group vs 9 for AOI-group; $\mathrm{p}=0.001$ ) (Table $4)$. The median time from remission to relapse was 32.0 months (IQR, 6.0 to 62.0 months) for AOI+ group and 31.0 months (IQR, 10.8 to 60.3 months) for AOI- group $(p=0.891)$. There was no significant difference in the cumulative relapse rate $(\mathrm{p}=0.759)$ between groups.

\section{DISCUSSION}

Incidental terminal ileal lesions are commonly found during colonoscopy. Although terminal ileitis can be caused by Crohn's disease, it is commonly found in benign conditions such as drug-induced (nonsteroidal antiinflammatory drugs) enteropathy, alcohol-induced intestinal mucosal injury, or infectious enteritis; and it can occur even in the healthy population. These lesions usually subside without treatment, and are considered benign. ${ }^{20}$ They can also be detected in patients with UC, especially as a form of backwash ileitis. Although there have been many reports about backwash ileitis and its clinical significance, nonspecific terminal ileal inflammation and its clinical significance in UC, especially in remission, has seldom been investigated.

We selected patients with UC who were in clinical remission, but excluded terminal ileal lesions possibly caused by backwash ileitis that can occur in moderate-to-severe disease states of UC (Mayo endoscopic score of 2 or 3 ). ${ }^{21,25}$ This study aimed to evaluate the clinical significance and prognostic value of terminal ileal lesions in patients with $\mathrm{UC}$ in clinical remission. In other words, we tried to determine the clinical significance of these incidental lesions,

Table 4. Baseline Patient Characteristics According to Appendiceal Orifice Lesions

\begin{tabular}{|c|c|c|c|}
\hline \multirow{2}{*}{ Characteristics } & \multicolumn{2}{|c|}{ Appendiceal orifice lesion } & \multirow{2}{*}{$p$-value } \\
\hline & Positive (n=107) & Negative ( $n=97)$ & \\
\hline Age at diagnosis, yr & $38.91 \pm 15.21$ & $34.78 \pm 13.96$ & 0.045 \\
\hline Age at the time of colonoscopy on remission, yr & $42.70 \pm 15.07$ & $41.04 \pm 14.52$ & 0.425 \\
\hline Height, cm & $165.90 \pm 8.68$ & $167.1 \pm 9.20$ & 0.335 \\
\hline Weight, kg & $62.21 \pm 12.58$ & $62.77 \pm 13.15$ & 0.758 \\
\hline BMI, $\mathrm{kg} / \mathrm{m}^{2}$ & $22.45 \pm 3.25$ & $22.42 \pm 4.07$ & 0.963 \\
\hline Sex & & & 0.130 \\
\hline Male & $61(57.0)$ & $45(46.4)$ & \\
\hline Female & $46(43.0)$ & $52(53.6)$ & \\
\hline Smoking at diagnosis & & & 0.119 \\
\hline Never-smoker & $70(65.4)$ & 73 (75.3) & \\
\hline Former smoker & $28(26.2)$ & $19(19.6)$ & \\
\hline Current smoker & $9(8.4)$ & $5(5.2)$ & \\
\hline Family history & $8(7.5)$ & $6(6.2)$ & 0.716 \\
\hline Appendectomy & 0 & $9(9.3)$ & 0.001 \\
\hline Time from diagnosis to remission, mo & $37.0(19.0-61.0)$ & $60.0(36.5-101.5)$ & $<0.001$ \\
\hline Initial disease extent & & & 0.003 \\
\hline AOI only & $2(1.9)$ & 0 & \\
\hline E1 & 57 (53.3) & $36(37.1)$ & \\
\hline E2 & $32(29.9)$ & $33(34.0)$ & \\
\hline E3 & $16(15.0)$ & $28(28.9)$ & \\
\hline Mayo score at remission & & & 0.688 \\
\hline 0 & $51(47.7)$ & $46(47.4)$ & \\
\hline 1 & $39(36.4)$ & $41(42.3)$ & \\
\hline 2 & $11(10.3)$ & 8 (8.2) & \\
\hline 3 & $6(5.6)$ & $2(2.1)$ & \\
\hline Mayo endoscopic score & & & 0.151 \\
\hline 0 & $68(63.6)$ & $52(53.6)$ & \\
\hline 1 & $39(36.4)$ & $45(46.4)$ & \\
\hline
\end{tabular}

Data are presented as mean $\pm \mathrm{SD}$, number $(\%)$, or median (interquartile range).

$\mathrm{BMI}$, body mass index; AOI, appendiceal orifice inflammation; E1, ulcerative proctitis; E2, left-sided ulcerative colitis; E3, extensive ulcerative colitis. 
given that the pathogenesis of TI lesions in patients with UC has not been elucidated (possibility of a latent focus of a future inflammation, a residual inflammation of a previous disease, or just incidental findings). ${ }^{35-37}$ Although a previous report suggested that the pathogenesis of terminal ileitis in UC might be different from that of healthy population, more studies are needed to elucidate its pathogenesis. ${ }^{35}$

In our study, it is noteworthy that never-smokers were more likely to have terminal ileal lesions $(\mathrm{p}=0.005)$. Nonsmoking patients are known to have higher relapse rates; however, recent meta-analyses recommended smoking cessation for patients with UC to improve their general health, regardless of the positive effects of smoking on UC. ${ }^{7,38}$ It remains unknown why TI+ lesions were prevalent in non-smokers. Although TI findings did not predict future relapse, it is still probable that TI lesions are strongly related to the pathophysiology of UC. Additionally, the time from diagnosis to the performance of colonoscopy at remission (months) was significantly longer in $\mathrm{TI}+$ group than in TI- group (63.0 months [37.0 to 109.0] for TI+ group and 38.0 months [21.0 to 66.0] for TI- group, respectively, $\mathrm{p}<0.001)$. Longer disease durations of UC were associated with TI+ lesions in our study. Therefore, longer durations of UC might be related to a possible dormant or remnant inflammatory source in the TI. Interestingly, a greater proportion of patients with a Mayo endoscopic score of 1 were included in TI+ group than in TI- group ( $58 \%$ in TI+ group vs $32.6 \%$ in TI- group, $\mathrm{p}=0.001$ ). The cumulative relapse incidence was not significantly different between the groups in our study, although it is supposed that a severe endoscopic inflammation at baseline predicts worse clinical outcome. ${ }^{39}$ Of interest, in TI+ group, followup colonoscopic examinations were performed in $38 \mathrm{pa}$ tients (55.1\%). Among them, disease extension was found in only two patients (E2 to E3 and E1 to E2). It appears that TI inflammation does not have any prognostic value in predicting disease extension. The Cox regression analysis revealed that advanced age at the time of colonoscopy during remission was related to lower risk of future relapse (adjusted HR, 0.966; 95\% CI, 0.937 to 0.995; $\mathrm{p}=0.021$ ). This finding was consistent with previous findings that a more advanced age was significantly associated with decreased relapse episodes. ${ }^{7}$ Univariate and multivariate Cox regression analyses did not show any meaningful relationship between TI lesions and future relapse.

In our study, AOI had different characteristics compared to TI lesions. AOI was more common in patients with advanced age, an E1 disease extent on the Mayo classification, and shorter duration from initial diagnosis of $\mathrm{UC}(\mathrm{p}=0.045, \mathrm{p}=0.003$, and $\mathrm{p}<0.001$, respectively). Patients with AOI were less likely to have had appendectomy in the past ( $\mathrm{p}=0.001$ ). There have been many reports that have investigated the association between AOI and UC, most of which reported no significant prognostic association between the AOI and UC. ${ }^{14,15,40,41}$ In this study, we similarly could not find any clinical meaning of the AOI as a prognostic factor in accordance with previous studies.

In addition, in TI+ group, 10 patients (14.5\%) had AOI, whereas in TI- group, 98 patients $(71.9 \%)$ had AOI $(\mathrm{p}<0.001)$. This seems to show that TI lesions have a different pathophysiology compared with that of AOI. In other words, they are not likely to be closely associated, although they are anatomically very close.

In this study, neither TI lesions nor AOI were significantly related to future relapse (Fig. 3). Therefore, in a clinical situation, observation rather than aggressive management or short-term full colonoscopy might be recommended.

As far as we know, our study is the first to follow the clinical course of terminal ileal lesions in patients with UC in clinical remission. In contrast to our study, Hamilton et al. ${ }^{25}$ prospectively compared the frequency and characteristics of terminal ileitis in 72 patients with UC to those of 90 healthy controls $(n=16[22 \%]$ vs $n=4[4 \%]$; $\mathrm{p}<0.001)$. They reported that terminal ileitis was more frequently found in patients with UC (more than one-fifth) compared to healthy population. The study indicated that the prevalence of terminal ileitis in patients with UC was higher than expected, although the sample size was small and prognostic data was limited. Our study offers some clinically meaningful significance in that we investigated a larger volume of terminal ileal lesions and showed the prognostic value of TI lesions in UC for the first time.

There were some limitations in our study. First, the data collection depended on retrospective electronic medical charts and image reviews. Second, we included patients with mild degrees of remnant UC on endoscopic examination (Mayo endoscopic score 1). Although we analyzed the patients with UC not only in endoscopic remission but also in clinical remission, it would be still valuable to investigate the prognostic significance of TI lesions in patients in remission based on clinical symptoms. Third, in real clinical practice, not all patients with UC in clinical remission undergo colonoscopy, which might have been associated with a selection bias in our study. Lastly, the presence or absence of terminal ileal lesions at the time of diagnosis of UC was not known in this study. In clinical practice, sigmoidoscopy is performed more frequently for the diagnosis of UC and other inflammatory bowel diseases, as it is not easy to complete a full colonoscopy during an acute flare phase of diseases. Although it is reasonable to elucidate the clinical features and natural history of terminal ileal lesions from 
the initial diagnosis, it is not practical in clinical situations.

In conclusion, for patients with UC who were in clinical remission, TI lesions and AOI had no significant clinical value for future relapse.

\section{CONFLICTS OF INTEREST}

J.H.C. and S.J.P. are editorial board members of the journal but were not involved in the peer reviewer selection, evaluation, or decision process of this article. No other potential conflicts of interest relevant to this article were reported.

\section{AUTHOR CONTRIBUTIONS}

Study concept: J.Y., J.H.C. Data analysis and collection: J.Y., S.J.P., J.P., T.I.K., W.H.K., J.H.C. Statistical analysis: J.Y., J.H.C. Writing - original draft: J.Y., J.H.C. Writing - review and editing: J.Y., E.A.K., S.J.P., J.J.P., T.I.K., W.H.K., J.H.C.

\section{ORCID}

Jongwook Yu Jihye Park Eun Ae Kang Soo Jung Park Jae Jun Park Tae Il Kim Won Ho Kim Jae Hee Cheon https://orcid.org/0000-0001-7489-2222 https://orcid.org/0000-0001-5253-2138 https://orcid.org/0000-0003-0220-937X https://orcid.org/0000-0003-0699-6809 https://orcid.org/0000-0001-9974-1658 https://orcid.org/0000-0003-4807-890X https://orcid.org/0000-0002-5682-9972 https://orcid.org/0000-0002-2282-8904

\section{REFERENCES}

1. McGovern DP, Gardet A, Törkvist L, et al. Genome-wide association identifies multiple ulcerative colitis susceptibility loci. Nat Genet 2010;42:332-337.

2. Cleynen I, Boucher G, Jostins L, et al. Inherited determinants of Crohn's disease and ulcerative colitis phenotypes: a genetic association study. Lancet 2016;387:156-167.

3. Triantafillidis JK, Merikas E, Georgopoulos F. Current and emerging drugs for the treatment of inflammatory bowel disease. Drug Des Devel Ther 2011;5:185-210.

4. Kim B, Park SJ, Hong SP, Kim TI, Kim WH, Cheon JH. Proximal disease extension and related predicting factors in ulcerative proctitis. Scand J Gastroenterol 2014;49:177-183.

5. Lee HJ, Jung ES, Lee JH, et al. Long-term clinical outcomes and factors predictive of relapse after 5-aminosalicylate or sulfasalazine therapy in patients with mild-to-moderate ulcerative colitis. Hepatogastroenterology 2012;59:1415-1420.

6. Colombel JF, D’haens G, Lee WJ, Petersson J, Panaccione R. Outcomes and strategies to support a treat-to-target approach in inflammatory bowel disease: a systematic review. J Crohns Colitis 2020;14:254-266.

7. Höie O, Wolters F, Riis L, et al. Ulcerative colitis: patient characteristics may predict 10 -yr disease recurrence in a European-wide population-based cohort. Am J Gastroenterol 2007;102:1692-1701.

8. Frøslie KF, Jahnsen J, Moum BA, Vatn MH; IBSEN Group. Mucosal healing in inflammatory bowel disease: results from a Norwegian population-based cohort. Gastroenterology 2007;133:412-422.

9. Beaugerie L, Massot N, Carbonnel F, Cattan S, Gendre JP, Cosnes J. Impact of cessation of smoking on the course of ulcerative colitis. Am J Gastroenterol 2001;96:2113-2116.

10. Kim JH, Cheon JH, Park Y, et al. Effect of mucosal healing (Mayo 0) on clinical relapse in patients with ulcerative colitis in clinical remission. Scand J Gastroenterol 2016;51:10691074.

11. Wang J, Nakamura TI, Tuskey AG, Behm BW. Polypharmacy is a risk factor for disease flare in adult patients with ulcerative colitis: a retrospective cohort study. Intest Res 2019;17:496-503.

12. Matsushita M, Fukata N, Omiya M, Nishio A, Seki T, Okazaki K. Pathophysiology of the appendix in ulcerative colitis. Am J Gastroenterol 2018;113:622.

13. Anzai H, Hata K, Kishikawa J, et al. Appendiceal orifice inflammation is associated with proximal extension of disease in patients with ulcerative colitis. Colorectal Dis 2016;18:O278-O282.

14. Park SH, Loftus EV Jr, Yang SK. Appendiceal skip inflammation and ulcerative colitis. Dig Dis Sci 2014;59:2050-2057.

15. Byeon JS, Yang SK, Myung SJ, et al. Clinical course of distal ulcerative colitis in relation to appendiceal orifice inflammation status. Inflamm Bowel Dis 2005;11:366-371.

16. McHugh JB, Appelman HD, McKenna BJ. The diagnostic value of endoscopic terminal ileum biopsies. Am J Gastroenterol 2007;102:1084-1089.

17. Jeong SH, Lee KJ, Kim YB, Kwon HC, Sin SJ, Chung JY. Diagnostic value of terminal ileum intubation during colonoscopy. J Gastroenterol Hepatol 2008;23:51-55.

18. Sayilir A, Kurt M, Kekilli M, et al. Diagnostic value of terminal ileum biopsy in chronic diarrhea with normal endoscopic appearance. J Dig Dis 2011;12:188-192.

19. Börsch G, Schmidt G. Endoscopy of the terminal ileum. Lancet 1984;2:573-574.

20. Chang HS, Lee D, Kim JC, et al. Isolated terminal ileal ulcerations in asymptomatic individuals: natural course and clinical significance. Gastrointest Endosc 2010;72:1226-1232. 
21. Bojic D, Markovic S. Terminal ileitis is not always Crohn's disease. Ann Gastroenterol 2011;24:271-275.

22. Sands BE. From symptom to diagnosis: clinical distinctions among various forms of intestinal inflammation. Gastroenterology 2004;126:1518-1532.

23. Lewis JD, Chuai S, Nessel L, Lichtenstein GR, Aberra FN, Ellenberg JH. Use of the noninvasive components of the Mayo score to assess clinical response in ulcerative colitis. Inflamm Bowel Dis 2008;14:1660-1666.

24. Achieving clinical response and remission in moderateto-severe ulcerative colitis with golimumab. Gastroenterol Hepatol (N Y) 2014;10:452-454.

25. Hamilton MJ, Makrauer FM, Golden K, et al. Prospective evaluation of terminal ileitis in a surveillance population of patients with ulcerative colitis. Inflamm Bowel Dis 2016;22:2448-2455.

26. Goldstein NS. Isolated ileal erosions in patients with mildly altered bowel habits: a follow-up study of 28 patients. Am J Clin Pathol 2006;125:838-846.

27. Turner D, Griffiths AM, Mack D, et al. Assessing disease activity in ulcerative colitis: patients or their physicians? Inflamm Bowel Dis 2010;16:651-656.

28. Manginot C, Baumann C, Peyrin-Biroulet L. An endoscopic Mayo score of 0 is associated with a lower risk of colectomy than a score of 1 in ulcerative colitis. Gut 2015;64:1181-1182.

29. O'Connor A, Ford AC. Poor correlation between patientreported and endoscopic components of the Mayo score in ulcerative colitis. Gastroenterology 2016;150:1037-1039.

30. Fukuda T, Naganuma M, Sugimoto S, et al. Efficacy of therapeutic intervention for patients with an ulcerative colitis Mayo endoscopic score of 1. Inflamm Bowel Dis 2019;25:782-788.

31. Satsangi J, Silverberg MS, Vermeire S, Colombel JF. The Montreal classification of inflammatory bowel disease: controversies, consensus, and implications. Gut 2006;55:749753.
32. Barreiro-de Acosta M, Vallejo N, de la Iglesia D, et al. Evaluation of the risk of relapse in ulcerative colitis according to the degree of mucosal healing (Mayo 0 vs 1): a longitudinal cohort study. J Crohns Colitis 2016;10:13-19.

33. D'Haens G, Sandborn WJ, Feagan BG, et al. A review of activity indices and efficacy end points for clinical trials of medical therapy in adults with ulcerative colitis. Gastroenterology 2007;132:763-786.

34. Lobatón T, Bessissow T, De Hertogh G, et al. The modified Mayo endoscopic score (MMES): a new index for the assessment of extension and severity of endoscopic activity in ulcerative colitis patients. J Crohns Colitis 2015;9:846-852.

35. Yamamoto T, Maruyama Y, Umegae S, Matsumoto K, Saniabadi AR. Mucosal inflammation in the terminal ileum of ulcerative colitis patients: endoscopic findings and cytokine profiles. Dig Liver Dis 2008;40:253-259.

36. Patil DT, Odze RD. Backwash is hogwash: the clinical significance of ileitis in ulcerative colitis. Am J Gastroenterol 2017;112:1211-1214.

37. Haskell H, Andrews CW Jr, Reddy SI, et al. Pathologic features and clinical significance of "backwash" ileitis in ulcerative colitis. Am J Surg Pathol 2005;29:1472-1481.

38. To N, Ford AC, Gracie DJ. Systematic review with metaanalysis: the effect of tobacco smoking on the natural history of ulcerative colitis. Aliment Pharmacol Ther 2016;44:117126.

39. Reinisch W, Reinink AR, Higgins PD. Factors associated with poor outcomes in adults with newly diagnosed ulcerative colitis. Clin Gastroenterol Hepatol 2015;13:635-642.

40. Matsumoto T, Nakamura S, Shimizu M, Iida M. Significance of appendiceal involvement in patients with ulcerative colitis. Gastrointest Endosc 2002;55:180-185.

41. Yang SK, Jung HY, Kang GH, et al. Appendiceal orifice inflammation as a skip lesion in ulcerative colitis: an analysis in relation to medical therapy and disease extent. Gastrointest Endosc 1999;49:743-747. 